\title{
Два подхода к построению логики ${ }^{1}$
}

\author{
В. И. ШАЛАК
}

\begin{abstract}
The aim of this paper is a comparative analysis of two conceptually different approaches to the construction of logic. The first approach, going from Aristotle, called traditional, the second can be called semiotic.
\end{abstract}

Ключевые слова: основания логики, онтологические предпосылки, эпистемические предпосылки, семиотика

Целью настоящей работы является сравнительный анализ двух концептуально различных подходов к построению логики. Первый подход, идущий от Аристотеля, назовем традиционным, второй - семиотическим.

Прежде чем приступать к анализу, уточним понимание логики, которого мы будем придерживаться.

«Логика - это нормативная наука о формах и приемах интеллектуальной познавательной деятельности, осуществляемой с помощъю языка» [2, с. 9].

Важной чертой логики является нормативность. Смысл ее в следующем:

«Задача логики состоит в том, чтобы ответить на вопрос: как мы должны мыслить, если хотим достичь чели познавательного прочесса - получить адекватные знания об исследуемых обгектах. Логика, таким образом, является наукой не о сущем, а о должсном, наукой нормативной. Она вырабатывает нормы, критерии правильности осуществления интеллектуальных прочедур, формируя тем самым

\footnotetext{
${ }^{1}$ Работа выполнена при поддержке РГНФ, грант № 11-03-00761а.
} 
некий канон, стандарт, идеал, следование которому является необходимым условием успешного осуществления научной и вообще любой рачиональной деятельности» [2, с. 19].

\section{1 Традиционный подход}

При традиционном подходе построение логики начинается с принятия ряда онтологических предпосылок. Эти предпосылки в самом общем виде содержат ответ на вопрос, как устроен мир. Они являются отправной точкой, из которой разворачивается построение логики как науки. Начальные онтологические предпосылки традиционного подхода заключались в том, что мир состоит из вещей и свойств, которыми эти вещи могут обладать.

Вслед за онтологическими принимаются определенные эпистемические предпосылки. Они говорят о том, каким образом знания о мире получают представление в языке. Основными языковыми единицами, выполняющими эту функцию, считаются предложения. Характер отношения предложений к явлениям окружающей реальности фиксируется в понятии истинности.

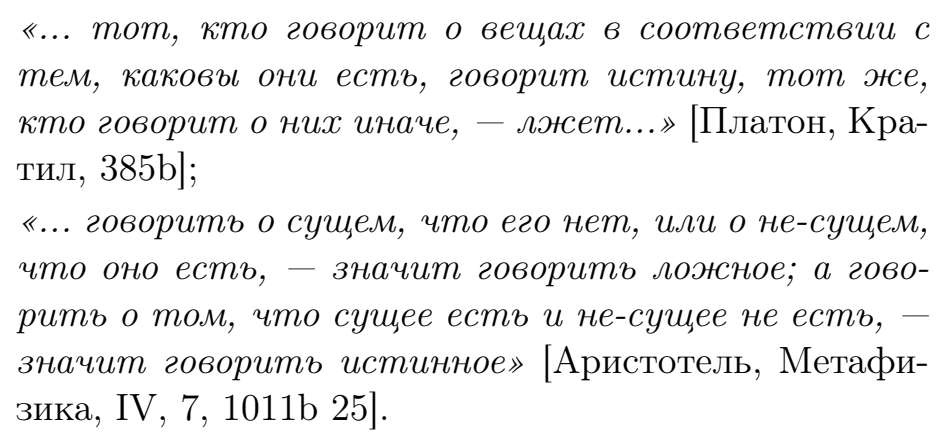

Свойство предложений быть истинными подчиняется законам, которые получили название законов логики. Изначально это были закон тождества, закон противоречия и закон исключенного третъего. Поскольку считалось, что субъектно-предикатная структура простых предложений воспроизводит объективно существующие отношения между вещами и свойствами, эти законы одновременно получили и онтологическую интерпретацию. Чтобы познание было возможным, законы мысли должны были следовать законам бытия. 
Третьей составляющей традиционного подхода к построению логики является формулировка целей познания.

Для Аристотеля целью познания было постижение вечных форм, существующих посредством отдельных вещей. Он разделял восходящее к Пармениду различение знания и мнения.

«Предмет знания и знание отличаются от предмета мнения и от мнения, ибо знание направлено на общее и основывается на необходимых положениях; необходимое же есть то, что не может быть иначе. Многое же хотя и истинно и существует, но может быть и иным. Ясно поэтому, что о нем нет науки» [Аристотель. Вторая Аналитика, 88 b 30-35].

Очевидно, что логика, в основу которой кладутся вполне конкретные предположения об устройстве мира, о характере его отражения в языке и целях познания, исторически ограничена и рано или поздно перестанет удовлетворять запросам реальной научной практики.

Логика Аристотеля с небольшими изменениями просуществовала вплоть до XIX в., когда наступила пора пересмотра ее оснований. В науке, и в первую очередь в математике, к тому времени были получены впечатляющие результаты, но они не имели логического обоснования. Была осознана ограниченность онтологии вещей и свойств, и на смену ей пришла онтология вещей и отношений, в которых эти вещи могут находиться. Свойства стали рассматривать как частный случай отношений. Было введено понятие пропозициональных функций, аргументами которых являются наборы индивидов, а значениями - Истина или Ложь. Это, в свою очередь, потребовало отказаться от жесткого параллелизма логических структур и структур естественного языка. Новая удобная символика позволила приступить к созданию искусственных языков. Многие математические теории получили строгое логическое обоснование. Если бы этим были удовлетворены все потребности реформы логики, то она еще долго сохраняла бы за собой звание канона. Однако ее дальнейшее развитие носило драматический характер.

Пророческими оказались слова Н.А. Васильева, сказанные им в 1912 г.: 
«В логике мы присутствуем при падении Великой Китайской стены: „эллинской и средневековой логики“. Мы присутствуем при неуклонном созидании „логики нового времени" творческими усилиями нескольких поколений. [...] Нужно расширить ее предель, удостоверитъся в бесконечности возможных логических систем. Тот, кто удостоверится в этом, будет испьтывать ощущения Джсордано Бруно, когда впервые в его воображснии предстала бесконечность физической Вселенной, когда разлетелись вдребезги хрустальные сферы неба. Все современное движение в логике есть восстание против Аристотеля, медленно, шаг за шагом идет это восстание то в одном пункте, то в другом. Трудно предсказывать будущее» [3, с. 123].

Интуитивно прозрачная онтология вещей и свойств оказалась логически невозможной. Предположение о том, что каждому свойству соответствует совокупность вещей им обладающих, вело к противоречию. В качестве альтернативных онтологий были предложены теория типов и несколько вариантов теории множеств. Выбор какой-либо из них зависел от личных предпочтений исследователя или соображения целесообразности для решения той или иной задачи. В результате появилось несколько математик.

Оценки отношения предложений языка к познаваемой реальности в терминах Истины или Лжи также подверглись критическому пересмотру. В современной логике не ограничиваются двумя истинностными значениями. Количество возможных значений достигло континуума. Придать им какой-то содержательный смысл практически невозможно. Появились логики с провалами истинностных значений, противоречивыми и нечеткими значениями. Понятие истинностного значения стало играть в логике все более и более техническую роль.

Н.А. Васильев, вводя различие эмпирической логики и металогики, отказался от обязательной онтологической интерпретации предложений языка. Законы мысли могут уже не следовать законам бытия. В результате варьирования логических законов не осталось ни одного, который не был бы отброшен в той или 
иной системе логики. Как и в случае с неевклидовой геометрией, то, что Н.А. Васильев называл воображаемой логикой, обрело реальность. Самый известный пример - логика квантовой механики.

Изменились и цели познания. Нас окружает изменчивый мир, наши ресурсы ограничены, мы более не надеемся познать абсолютную истину. Это также налагает отпечаток на допустимые способы рассуждений и получаемые выводы.

В результате всех трансформаций логика потеряла свой нормативный характер. Она перестала быть «каноном, стандартом, идеалом, следование которому является необходимым условием успешного осуществления научной и вообще любой рациональной деятельности». Окружающая реальность распалась на множество фрагментов, каждому из которых, если следовать традиционному подходу, соответствует своя логика. Вступая в сферу непознанного, мы уже не можем быть уверены в том, каким правилам должны следовать наши рассуждения, чтобы предохранить нас от заблуждений.

Закономерным итогом эволюции традиционного подхода явилась универсальная логика Ж.И. Безьё. Это уже не очередной свод логических законов, а построенная по аналогии с универсальной алгеброй теория всех возможных логик. Ж.И. Безьё сравнил ее с ящиком инструментов, используемых при построении конкретных логических систем для конкретных проблемных областей. Искусство таких построений схоже с приготовлением майонеза, когда из различных ингредиентов, временных, деонтических, эротетических и др. операторов, получают нечто пригодное для анализа и проведения конкретных типов рассуждений [5].

Из науки о должном логика превратилась в науку о возможном.

\section{2 Семиотический подход}

Закономерно задаться вопросом, возможен ли другой подход к построению логики, который, пусть в иных формах, но позволил бы сохранить за ней роль универсального инструмента интеллектуального познания, роль «нормативной науки о формах и приемах интеллектуальной познавательной деятельности, осуществляемой с помощью языка»? 
Обратим внимание на очевидный факт, что добытые людьми знания всегда получали и будут получать оформление в языке совершенно независимо от того, к какой области реальности они относятся. Это справедливо для знаний о внутреннем мире человека и знаний об окружающей нас физической реальности. Это справедливо для знаний о мире квантово-механических явлений, для знаний о макромире и для космологических теорий о происхождении и эволюции вселенной. Какие бы дополнительные предположения об устройстве мира ни принимались, знания все равно будут получать представление в языке. Это является необходимым условием их накопления и передачи от поколения к поколению. Не может существовать единственного языка, который был бы пригоден для этих целей и всегда сохранял это качество. Если мы хотим, чтобы логика действительно стала нормативной наукой, мы должны отвлечься от всякой специфики конкретных языков и взглянуть на них лишь как на абстрактные знаковые системы. Именно знаковая природа языка делает его универсально применимым для представления знаний о любой реальности и о любом ее фрагменте. Но содержится ли в столь абстрактно понимаемом языке хоть что-то относящееся к логике?

Первая зацепка, которую мы обнаруживаем, заключается в том, что язык как абстрактная знаковая система устроен иерархически.

«Язык представляет собой систему и единое целое. Он организуется как упорядоченный набор различимых и служсащих различению "знаков", которые обладают свойством разлагаться на единичь низшего порядка и соединяться в единицы более сложные. Эта большая структура, включающая в себя меньшие структуры нескольких уровней, и придает форму содержанию мысли. [...] Языковая форма является тем самым не только условием передачи мысли, но прежде всего условием ее реализации. Мы постигаем мысль уже оформленной языковыми рамками» [1, c. 105].

Выражения языка, рассматриваемые как знаки, призваны выполнять функцию быть представителями различных объектов 
мысли. Это все что о них можно сказать. О конкретной природе объектов мысли заранее ничего не известно. Это могут быть вещи, свойства, функции, отношения и т.д. Их объединяет лишь то, что все они являются объектами мысли и в этом смысле совершенно равноправны. Разбиение объектов мысли на категории возможно, но произойти это может только в результате познавательной деятельности субъекта познания.

Язык логического субъекта, еще не приступившего к процессу познания, в информационном смысле пуст. Он не содержит даже констант для представления конкретных объектов мысли, поскольку ничто еще не поименовано. Чтобы иметь возможность говорить о произвольных выражениях языка, нам потребуются переменные, пробегающие по этим выражениям. Для представления иерархической структуры выражений языка понадобится синтаксическая операция, позволяющая на самом абстрактном уровне из более простых выражений языка строить более сложные. Можно показать, что, с математической точки зрения, достаточно ограничиться всего лишь одной бинарной синтаксической операцией. Для ее представления удобно использовать скобки. В этом случае построение сложных выражений языка будет производиться по схеме:

Если $A$ и $B$ - выражения языка, то $(A B)$ также выражение языка.

Но логика не сводится к одним лишь выражениям языка. Она предполагает существование правил рассуждений - рационально обоснованных переходов от одних выражений языка к другим. Каким может быть обоснование таких правил в языке как знаковой системе?

Великая сила языка как инструмента познания заключается в том, что он позволяет нам оперировать объектами внеязыковой реальности на знаковом уровне. Это оперирование можно представить как цепочку переходов от одних выражений языка к другим. В процессе манипулирования языковыми выражениями связь между ними и объектами внеположной реальности не должна теряться. Это является необходимым условием правильности нашей языковой деятельности. Одни переходы между выражениями обосновываются уже известными свойствами 
соотнесенных им объектов мысли. Другие - получают обоснование благодаря свойствам самого языка. В самом общем виде отношение следования между выражениями языка как знаками можно определить следующим образом:

Из посылок $\Sigma=\left\{B_{1}, \ldots, B_{n}\right\}$ следует выражение $A$, если и только если существует правило $R$, которое позволяет на основании значений посылок $\Sigma$ определить значение выражения $A$.

Сформулированная таким образом идея рассуждения не нуждается в уточнении того, о каких конкретно языковых выражениях идет речь, какова природа соответствия между этими выражениями и внеязыковой реальностью, что представляет собой эта реальность.

Определенное нами отношение следования обладает рядом свойств.

Если выражение А уже содержится среди посылок $\Sigma$, значения которых известны, то тем самым известно и значение $A$.

Следующее свойство позволяет строить цепочки переходов от одних выражений к другим. Рассуждения могут состоять из нескольких шагов, если между ними имеется связь.

Если существует правило $R$, которое позволяет на основании значений посылок $\Sigma$ определить значение выражения $A$, и существует правило $Q$, которое позволяет на основании значения $A$ определить значение выражсения $B$, то последовательное применение этих двух правил $R$ и $Q$ позволит на основании значений посылок $\Sigma$ определить значение $B$.

Справедливость этих двух свойств обоснована самим определением следования, но если ограничиться только ими, полученная в результате система рассуждений окажется слишком бедной. Она будет адекватна языку, содержащему лишь простые выражения. Мы пока что никоим образом не учли тот факт, что язык, наряду с простыми, содержит также сложные выражения, значения которых зависят от значений входящих в них более простых выражений. Если мы знаем значения выражений 
$A$ и $B$, то мы можем определить значение $(A B)$. Это свойство имеет место не по причине конкретной семантики, а по причине нашего понимания языка - что значения сложных выражений определяются значениями составляющих их частей. Когда мы формулируем в языке некое новое сообщение для передачи другим, мы уверены, что адресат поймет нами сказанное, поскольку и мы, и он пользуемся одним языком с одним и тем же синтаксисом, одной и той же семантикой. Это и является обоснованием следующего правила.

Если из посылок $\Sigma$ следует $A$, и из $\Sigma$ следует $B$, то из $\Sigma$ следует $(A B)$.

Очевидно, что перечисленные выше свойства не предполагают каких-либо онтологических или эпистемических допущений. Они базируются на абстрактных свойствах самого языка, а не на гипотетических свойствах окружающего мира.

Семиотический подход к построению логики этим не исчерпывается. В основе его дальнейшего развертывания лежат уже не свойства языка, а наблюдение за особенностями деятельности логического субъекта.

Ситуация, в которой некоторый объект выступает в роли знака, содержит три обязательных элемента: знак, означаемое и интерпретатора. Их взаимоотношение заключается в том, что знак представляет (репрезентирует, замещает) означаемое для интерпретатора. Такую ситуацию называют знаковой или $c е-$ миозисом. Роль интерпретатора в семиозисе играет логический субъект. Данная роль наделяет его правом расширять язык новыми знаками. Без этого никакое новое знание не могло бы получить представления в языке. Одни знаки он вводит в язык, чтобы репрезентировать те или иные явления окружающего мира или произвольные объекты мысли. Вследствие этого помимо логической они несут еще и внелогическую нагрузку. Другие знаки он вводит в язык без какого-либо обращения к внеположной реальности, и потому правила оперирования ими должны быть отнесены к логике.

Если имеется некоторое выражение языка, которое по какимто причинам представляет для логического субъекта особый интерес, он может запомнить его, воспользовавшись для этого операцией определения. 
«В логике определением (дефиницией) называют логическую прочедуру придания строго фиксированного смысла языковым выражениям (терминам языка)» $[2$, c. 197].

Одними из самых простых и распространенных являются явные определения. Они имеют следующую лингвистическую форму:

$$
A=\operatorname{def} B
$$

Левая часть этого выражения, обозначенная буквой $A$, называется определяемым (дефиниендумом), а правая часть, обозначенная буквой $B$, - определяющим (дефиниенсом). Символ

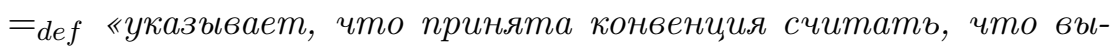
ражение $A$ означает то же самое, что и выражсние $B$ » $[2$, c. 201]. В левой части определения обязательно присутствует новый так называемый определяемый термин, который логический субъект вводит в свой язык, расширяя его.

Поскольку введение явных определений не влечет за собой принятия каких-либо предпосылок об окружающем мире, а является своего рода соглашением, которое логический субъект заключает с самим собой, расширим определение следования следующим образом.

Из посылок $\Sigma$ и определений $\Delta$ следует $A$, если и только если существует правило $R$, которое позволяет на основании определений $\Delta$ и значений посълок $\Sigma$ определить значение $A$.

Определяемый термин - это не просто новая константа языка. За ним закрепляется роль знака - представителя структуры дебиниенса определения. С того момента, когда принято определение, это становится личным знанием логического субъекта как интерпретатора знаковой системы. Поэтому отношение следования обладает следующим тривиальным свойством.

Если $T$ - определяемый термин, введенный одним из определений $\Delta$, а $\Sigma-$ множество посылок, то из $\Delta$ и $\Sigma$ следует $T$. 
Коль скоро левая и правая части определения означают одно и то же, этим можно пользоваться при построении рассуждений, свободно заменяя одно другим.

«Явные определения обладают одним замечательным свойством - определяемые и определяющие части могут в любом контексте замещаться друг на друга, то есть для них верно следующее правило:

$$
\frac{C={ }_{\text {def }} D, \quad K[C]}{K[D / C]}
$$

называемое правилом замены по дефиниции. ... Это правило позволяет использовать явные определения в прочессах дедуктивного вывода» [2, с. 207].

Обоснование данного правила считается настолько очевидным, что обычно его даже не приводят. С использованием правила замены часто строят свои рассуждения люди, даже не знакомые с тем, что такое логика.

Так как, согласно правилу замены, значение выражения $K[D / C]$ совпадает со значением $K[C]$, это позволяет нам сформулировать еще одно свойство следования.

Если из посылок $\Sigma$ и множества определений $\Delta$, содержащего $C={ }_{\text {def }} D$, следует выражение $K[C]$, то из $\Sigma$ и $\Delta$ будет следовать выражение $K[D / C]$.

Может показаться, что введение определений и правило их замены не дают ничего принципиально нового. Действительно, если рассматривать явные определения чисто экстенсионально, то значение левой части всего лишь совпадает со значением правой. Но дело в том, что помимо значений обе части явных определений обладают еще и структурой. Заменяя в ходе рассуждения определяемое на определяющее, логический субъект оперирует не со значениями выражений, а со сложными языковыми структурами, и в этом смысле переход, совершаемый от одного выражения к другому, на символьном уровне не является тождественным.

Существуют ли другие свойства отношения следования, которые пока что ускользнули от нашего внимания? На этот вопрос 
мы можем дать отрицательный ответ. Перечисленный нами набор свойств в математически строгом смысле является полным и потому может служить основанием логики, создаваемой на пути семиотического подхода.

В этой логике могут быть представлены любые эффективные рассуждения [4]. Интересной ее особенностью является то, что большинство известных логических систем теряют в ней свой прежний статус и превращаются в прикладные теории. В этом нет ничего удивительного, поскольку это является прямым следствием принимаемых в них онтологических и эпистемических предпосылок. В то же время некоторые из традиционно понимаемых теорий приобретают более высокий логический статус.

Если обратиться к истории науки, то в ней можно обнаружить попытки строить рассуждения в духе семиотического подхода. Прежде всего имеется в виду то, что уже в наше время получило не слишком удачное название рецептурной математики. Правила для вычисления площади прямоугольного участка земли по его сторонам, правила для вычисления объемов пирамид и т.д. служили семантическим обоснованием для перехода от одних выражений языка к другим. Рано или поздно такая практика потребовала бы выделения универсальных правил рассуждений, т.е. построения логики на основе семиотического подхода. Исторически, по причине быстрого распространения более успешной эллинистической культуры, этого не произошло, и логика стала развиваться по уже известному пути, что в конце концов привело к ее дроблению на множество частных методов и постепенной потере статуса философской дисциплины.

\section{Литература}

[1] Бенвенист Э. Категории мысли и категории языка // Общая лингвистика. Благовещенск: БГК им. И.А. Бодуэна де Куртенэ, 1998. С. 104-114.

[2] Бочаров B.А., Маркин В.И. Основы логики. Учебник. М.: Космополис, 1994.

[3] Васильев Н.А. Логика и металогика // Воображаемая логика. Избранные труды. М.: Наука, 1989. С. 94-123.

[4] Шалак В.И. Протологика: новый взгляд на природу логического // Дисс. ... докт. филос. наук. М., 2010.

[5] Beziau J-Y. 13 Questions about Universal Logic // Bulletin of the Section of Logic. V. 35, №2/3, 2006. P.133-150. 